\title{
Quantifying the Effects of Light Scattering in a Confocal Microscopy Imaging System
}

\author{
D. G. Pasternack*
}

* Dept. Environmental Medicine, Univ. Roch., 601 Elmwood Ave, Box EHSC, Roch., NY 14642

The challenge for imaging fluorophores in biological tissue is to eliminate contribution of fluorophores that are not within the focal plane. Confocal microscopy utilizes laser light to excite samples. A pinhole, located prior to the detector, restricts the detection of fluorophores to a thin "optical section", excluding fluorophores that are outside the plane of focus, preventing corruption of the focal plane [1]. However, biological tissue contains numerous intracellular organelles that scatter laser light on its path to the fluorophore of interest. The scattered light diffracts from the focal point and excites out-of-focus fluorophores. The resulting emission can also scatter, pass through the pinhole, and be detected leaving a reduction in fluorescence intensity of the intended target.

Since the excited out-of-focus fluorophores are of the same color as the fluorophores within the focal plane, deciphering the fraction of intensity in the captured image due to scattered light as compared to the target volume within tissue has not been feasible. From Mie Theory, we know that light will scatter from small particles that have a different refractive index than the medium they are in [2]. We hypothesized that creation of an in vitro system in which polystyrene beads served as scattering "organelles" would enable the analysis of the extent of confocal microscopy scattering.

To quantify the effects of light scattering, we constructed an in vitro model in which a red fluorophore embedded polymer rod was lowered into a fluorescein solution containing a concentration of either $0.1 \mu \mathrm{m}$ or $2 \mu \mathrm{m}$ diameter polystyrene beads. The beads served as scatterers and thus modeled intracellular organelles in tissue. By focusing on the red polymer rod at different depths within the solution, the green fluorescein represented the amount of scattered fluorescence detected. Imaging occurred with two magnifications on a confocal microscope constructed by the Optics Department of the University of Rochester [3].

Our results demonstrate that the angular distribution of scattered light depends on the size of the intracellular organelle. Organelles approximately $0.1 \mu \mathrm{m}$ in diameter have a large angular distribution of scattered light, whereas organelles that are $2 \mu \mathrm{m}$ in diameter have a small angular distribution. It is interesting to note that the $10 \mathrm{x}, 0.45 \mathrm{NA}$ objective (figure $2(\mathrm{C})$ ) shows more scattered light detected than the $4 x, 0.13$ NA objective (figure $2(\mathrm{E})$ ) with the $0.1 \mu \mathrm{m}$ beads.

\section{References}

[1] M. Minsky. Scanning 10: 128-138 (1988).

[2] S.L. Jacques, et al. Oreg Grad Inst (1998) http://omlc.ogi.edu/classroom/ece532/class3/mie.html. [3] C.E Bigelow, D.L. Conover, T.H. Foster. Opt. Lett. 28:695-697 (2003).

[4] This work represents a portion of the requirement for a master's degree through the Univ. Roch. Dept. Radiology and Institute of Optics. The author thanks the Institute of Optics for use of their microscope and the UR Pathology/Morphology Imaging Core for technical support. The aid of Dr. C.E. Bigelow and Dr. T.H. Foster is gratefully acknowledged. 


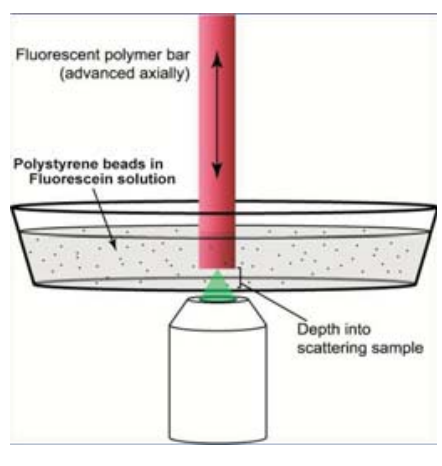

Figure 1. The flouroscein solution contains $2 \mathrm{ml}$ of fluoroscein along with a specificied concentration of polystyrene beads. There were two cases considered: a fluorescein solution with 2 micron diameter beads and a fluoroscein solution with 0.1 micron diamter beads. The concentrations of polystyrene beads was determined by the size of bead used. The objective collected the images at different focal depths. There were two objectives used: a 10x $0.45 \mathrm{NA}$ objective and a $4 \mathrm{x}, 0.13$ NA objective. The Red Polymer Rod was lowered into the fluorescein solution and the objective was focused on the bottom of the solution. We then used a controller to move the polymer rod every 100 microns until we were 1000 microns deep in the solution.
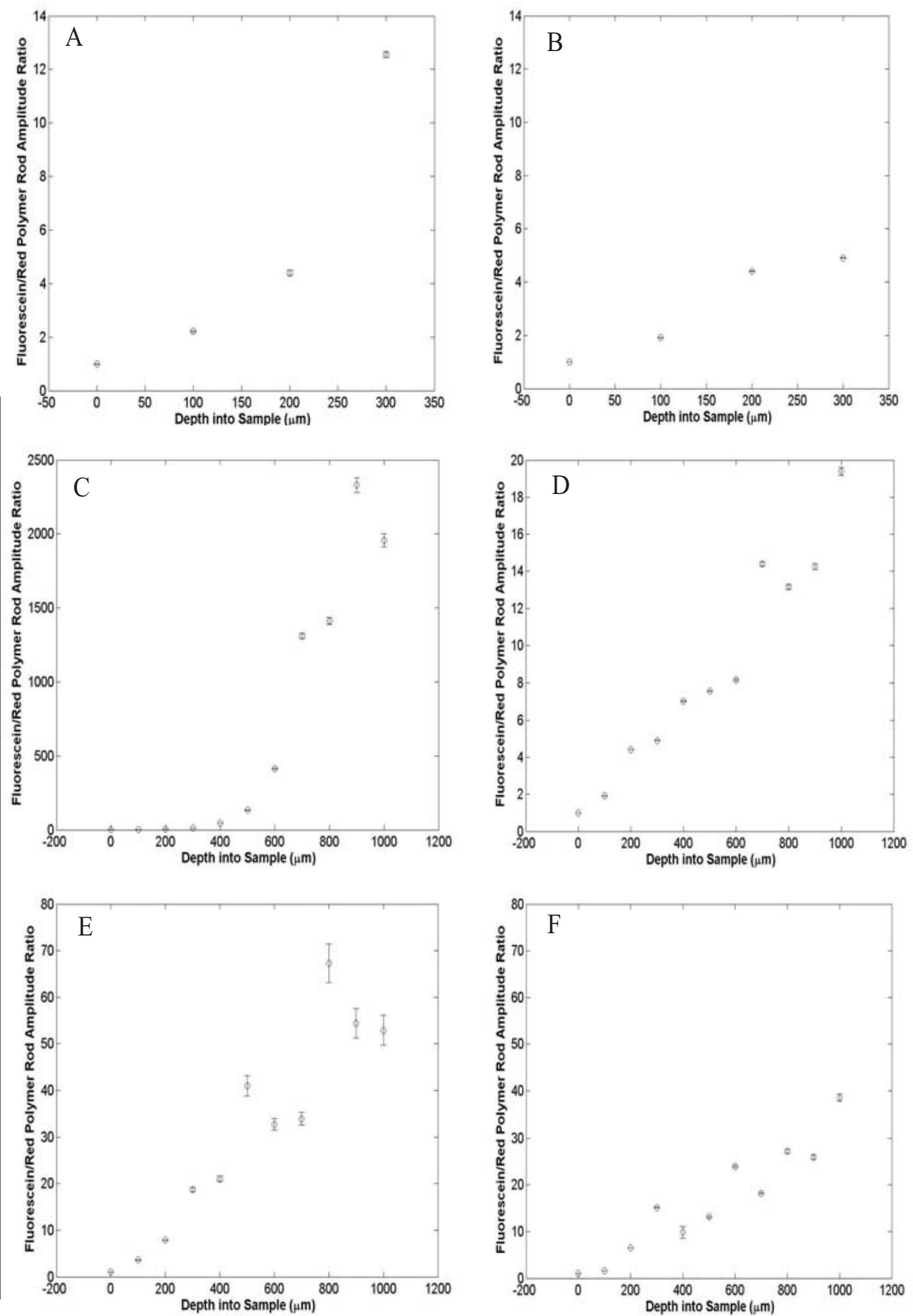

Figure 2. Ratio of fluorescein amplitudes to red polymer rod amplitudes as a function of depth into the solution. All the ratios at 0 micron depth are normalized to 1 . The error bars were calculated by the propagation of errors using the uncertainties in fluorophore amplitudes returned by the Singular Value Decompositon fitting algorithm. A) 10x, 0.45 NA objective with 0.1 micron diameter beads in solutions from 0 to 300 microns in depth. B) 10x, 0.45 NA objective with 2 micron beads in solution from 0 to 300 microns in depth. C) $10 x, 0.45$ NA objective with 0.1 micron diameter beads in soltuion from 0 to 1000 microns in depth. D) 10x, $0.45 \mathrm{NA}$ objective with 2 micron diameter beads in solution from 0 to 1000 microns in depth. E) 4x, 0.13 NA objective with 0.1 micron diameter beads in solution from 0 to 1000 microns in depth. F) 4x, 0.13 NA objective with 0.1 micron diameter beads in soltuion from 0 to 1000 microns in depth. 